\title{
Patient specific biomodel of the whole aorta - the importance of calcified plaque removal
}

\author{
A. Håkansson ${ }^{1}$, M. Rantatalo ${ }^{2}$, T. Hansen ${ }^{3}$ and A. Wanhainen ${ }^{4}$ \\ ${ }^{1}$ Divisions of Industrial Design, Luleå University of Technology, Luleå, Sweden \\ ${ }^{2}$ Division of Operation and Maintenance Engineering, Luleå University of Technology, Luleå, Sweden \\ ${ }^{3}$ Departments of Radiology, Uppsala University Hospital, Uppsala, Sweden \\ ${ }^{4}$ Department of Surgical Sciences, Section of Vascular Surgery, Uppsala University Hospital, Uppsala, Sweden
}

\section{Summary}

Background: The use of anatomical models produced by 3D printing technique (rapid prototyping, RP) is gaining increased acceptance as a complementary tool for planning complex surgical interventions. This paper describes a method for creating a patient specific replica of the whole aorta.

Methods: Computed tomography angiography (CTA) DICOM-data was converted to a three-dimensional computer aided design-model (CAD) of the inner wall of the aorta representing the lumen where the calcified plaque contribution was removed in a multi-step editing-manoeuvre. The edited CAD-model was used for creating a physical plaster model of the true lumen in a 3D-printer. Elastic and transparent silicon was applied onto the plaster model, which was then removed leaving a silicon replica of the aorta.

Results: The median (interquartile range) difference between diameters obtained from CTA- and RP plaster-model at 19 predefined locations was $0.5 \mathrm{~mm}(1 \mathrm{~mm})$ which corresponds to a relative median difference of $4.6 \%$ (7.0\%). The average wall thickness of the silicone model was $3.5 \mathrm{~mm}$. The elasticity property and performance during intervention was good with an acceptable transparency.

Conclusions: The integration of RP-techniques with CAD based reconstruction of $3 \mathrm{D}$-medical imaging data provides the needed tools for making a truly patient specific replica of the whole aorta with high accuracy. Plaque removal postprocessing is necessary to obtain a true inner wall configuration.

Key words: Biomodel, vascular model, rapid prototyping, aortic aneurysm

\section{Zusammenfassung}

Patientenspezifisches Biomodell der gesamten Aorta-Bedeutung der Entfernung von kalzifizierten Plaques

Hintergrund: Zur Planung komplexer chirurgischer Eingriffe wird zunehmend ein anatomisches Modell angefertigt, das mittels 3D Drucktechnik (schneller Prototypenbau, rapid prototyping, RP) erstellt wird. Wir beschreiben hier eine Methode, um eine patientengetreue Kopie der gesamten Aorta darzustellen.

Methoden: Computertomographisch generierte DICOM Daten einer Angiographie wurden in ein dreidimensionales computerassistiertes Designmodell (CAD) der inneren Aortenwand umgewandelt, wobei der Anteil an kalizifierten Plaques in mehreren Schritten entfernt wurde. Das bearbeitete CAD Modell wurde benützt, um ein Gipsmodell des wahren Lumens in einem 3D Drucker herzustellen. Elastisches transparentes Silikon wurde auf das Gipsmodell aufgetragen, das dann entfernt wurde, so dass eine Silikonkopie der Aorta verblieb.

Ergebnisse: Die mediane Differenz zwischen den Durchmessern von CTA und Gipsmodell an 19 Stellen betrug $0,5 \mathrm{~mm}$ (interquartil range, IR, $1 \mathrm{~mm}$ ), was einer relativen medianen Differenz von 4,6\% (IR 7,0\%) entspricht. Die durchschnittliche Wanddicke des Silikonmodells betrug 3,5 mm. Die elastischen Eigenschaften waren gut mit einer akzeptablen Transparenz.

Schlussfolgerungen: Die Integration von RP Techniken mit CAD basierten Rekonstruktionen von 3D Bilddaten gestattet es, eine patientengetreue Kopie der gesamten Aorta mit hoher Genauigkeit herzustellen. Eine rechnerische Entfernung von Plaques ist notwendig, um eine wahre Konfiguration der Gefäßwand zu bekommen.

\section{Introduction}

The use of anatomical models produced by rapid prototyping (RP) [3] is gaining increased acceptance as a complementary tool for planning complex surgical intervention [8]. Biomodels, in addition to standard imaging, improve measurement ac- curacy, reduce operating time, improve patients informed consent and are cost effective $[1,5,23]$. Furthermore, biomodels may facilitate manufacturing of custom-made endoprosthesis, be helpful in the development and evaluation of new treatment strategies and techniques, and for surgical practice and simulation
$[2,6,14,16]$. Today, biomodels are clinically mainly used in craniofacial-, skull base-, and pelvic surgery. Endovascular aortic aneurysm repair request a detailed preoperative planning in the three-dimensional space. However, the complexity of some aortic pathology, such as suprarenal- and thoracoabdominal aortic aneurysms, 
prevents the implantation of standard stentgrafts in these cases. An objective representation of the patient's anatomy, by means of a biomodel, may enhance the detailed preparation and fabrication of stentgrafts customized to patient anatomy. The implantability of the stentgraft can be verified and even rehearsed on the model too, and by using the model as a navigational aid during the intervention itself, operation time may be reduced. Biomodels may also be used in the development and evaluation of new devices and techniques, such as fenestrated- and branched stentgrafts, chimney-techniques, and in-situ fenestration. Finally, biomodels can serve as a complement to standard computer-based simulation programs for training in a realistic environment.

Traditionally, vascular replicas have been crafted from blown glass or cast in silicon rubber from human or animal blood vessels [4]. Neither technique fulfills the properties for a model to be clinically useful. Optimally, an aortic biomodel should include the whole aorta, i.e. the access vessels, the pathology itself with the proximal and distal landing zones, and sufficient length of all involved branches. Furthermore, it should be elastic and transparent. Elastic compliance allows the model wall to imitate the behaviour of the aortic wall during implantation, and transparency is essential to observe how the endovascular devices behave and how the implant fits. If the model is to be used under fluoroscopy, is should allow for good visibility of the devices used. However, most importantly an aortic replica must be accurate in terms of the internal surface of the vessel. Previous published studies describing human aorta replicas do not address the full problem with plaque contribution to the inner wall geometry when converting computed tomography (CT) data to computed aided design (CAD) and are mainly focused on smaller parts of the aorta $[7,10-12,18,20,21]$.

The integration of RP-techniques with CAD based reconstruction of three-dimensional medical imaging data provides the needed tools for making a truly patient specific replica of the aorta with high accuracy. The present work describes the design and development of an exact and clinically realistic replica of the whole aorta.

\section{Materials and methods}

\section{CT-angiography}

A 67-year old female patient with a thoracoabdominal aneurysm scheduled for repair was examined with a CT-angiography (CTA). A multidetector row CT system was used (Definition; Siemens Medical Systems, Forcheim, Germany). Helical CT-scans were done with $0.6 \mathrm{~mm}$ acquired slice thickness and reconstructed with $1 \mathrm{~mm}$ slices at $0.7 \mathrm{~mm}$ increment (space) from the lower aspect of the neck to the caudal aspect of the symphysis pubis, with the following parameters: $120 \mathrm{kV}$, 0.5 -second gantry rotation, and 150 reference $\mathrm{mAs}$ and CTDIvolume $9.67 \mathrm{mGy}$. The examination was done after intravenous administration of $100 \mathrm{~mL}$ nonionic iodine contrast material (Iopramide [300 mg of iodine per milliliter]; Berlex Laboratories, Wayne, NJ). The contrast material was injected through a 20 -gauge catheter inserted into an antecubital vein, at a rate of $4 \mathrm{~mL} / \mathrm{sec}$ with a power injector (Stellant Injection System, Medrad Inc., Warrendale, PA). Data acquisition began when a proximal aortic enhancement of $100 \mathrm{HU}$ was detected.

\section{Removal of calcified plaque}

The CTA-DICOM data was converted into an STL-file using Mimics-soft- ware (Materialize Group, Leuven, Belgium). By means of standard editing software tools, the contrast enhanced lumen, with its adherent atherosclerotic plaques, were separated from other body structures, leaving a lumen/plaque mask. With its high attenuation compared to the contrast enhanced lumen, the calcified plaque generates a transition area surrounding the plaque with a similar attenuation as the contrast. This is partly due to a partial volume effect that occurs when tissues of different attenuation are included in one voxel. The transition area does not occur in the case of more lipid rich plaques or thrombus due to the lower attenuation of these structures compared to the contrast. This transition area, previously surrounding the calcified plaque, will be interpreted as a part of the lumen and will therefore be included in a 3Dsurface reconstruction of the inner wall, resulting in a falsely larger inner diameter (Fig. 1a).

Thus, the calcified plaque had to be separated from the contrast manually, which was done in a multi-step editing-manoeuvre; 1) Using a higher attenuation threshold a plaque-mask was created (Fig. 1a). 2) The plaquemask was expanded to include the transition area surrounding the calcified plaque. The expanded plaquemask was then subtracted from the lumen/transition area-mask leaving a mask including only the lumen, however, too small in the direction of the calcified plaque (Fig 1b). 3) The mask was then expanded to the same extent as the plaque-mask, with the constraint that the mask can not exceed the size of the original lumen/ transition area-mask. This final operation resulted in a mask including only lumen (Fig. 1c), which was used to create a polygonal 3D-surface representation of the inner wall of the artery in STL-format suitable for RP. The multi-step editing-manoeuvre was performed in a software mode 


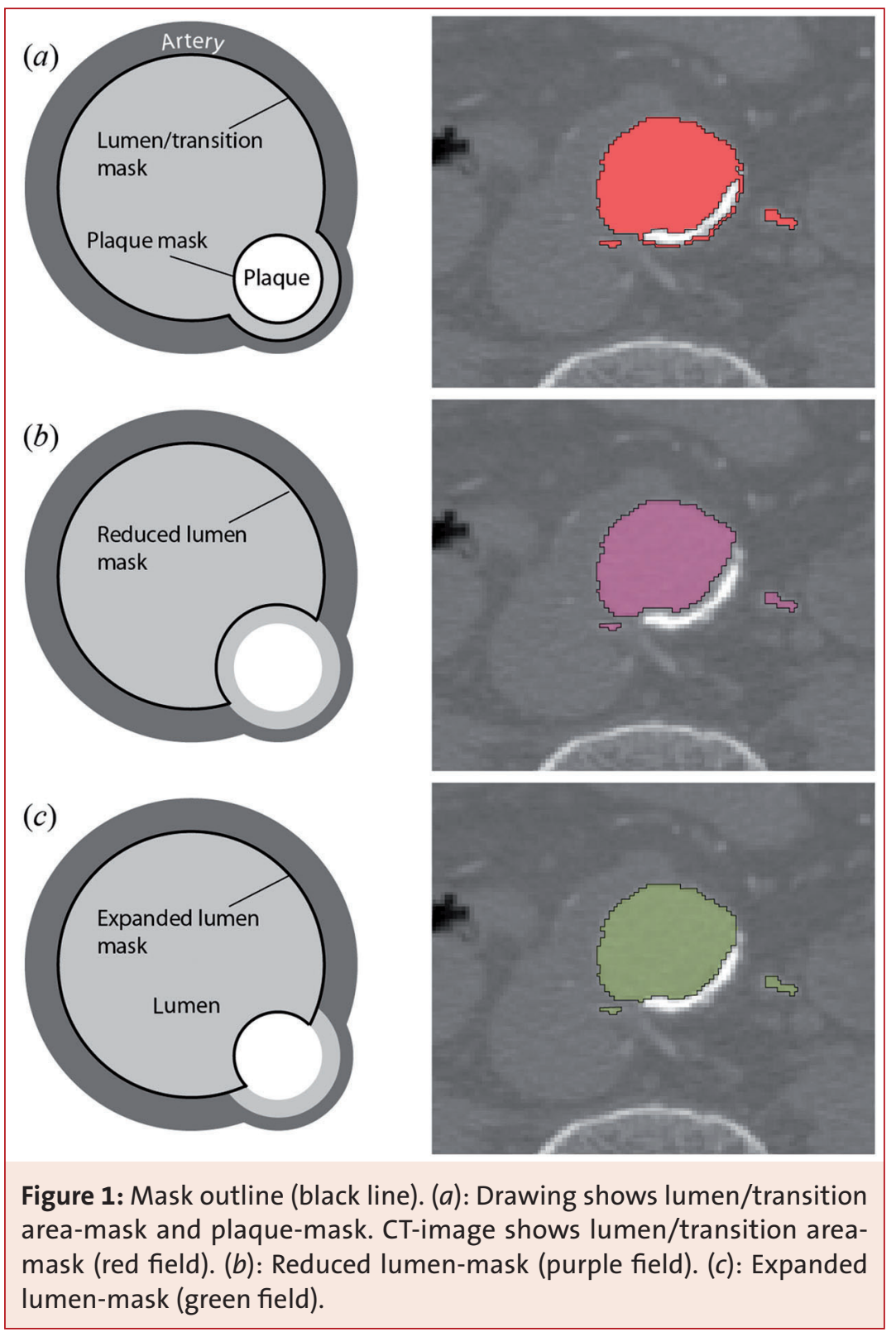

(26-connectivity) taking all spatial directions in account.

\section{Rapid prototyping}

The 3D-print was performed with a Z Corp 402 3D Printer (Z Corporation, Burlington, MA). To fit the model into the 3D-printer machine the STL-file was sliced into three segments using Geomagic Studio 3Dsoftware (Geomagic Inc., Research Triangle Park, NC). The three seg- ments were positioned on top of each other for printing in one single print, with a layer thickness of $0.1 \mathrm{~mm}$ using ZP 100 plaster powder (Z Corporation, Burlington, MA).

\section{Silicon modelling}

The three parts were linked and glued together and the surface was coated with thin layer of filler, to ensure the silicon to let loose properly. To create the final representation of the aorta, a number of layers of silicon were applied on to the surface of the RP-model. The silicon material used has an elongation at break of $400 \%$ and a tear strength of $25 \mathrm{~N} / \mathrm{mm}$. The intended aortic wall thickness was about $3 \mathrm{~mm}$ $[11,17]$. After curing of the silicon the RP-model (plaster core) was removed by breaking it into smaller pieces and flushing with water.

\section{Evaluation}

The accuracy of the model was reviewed by an experienced radiologist (TH) and an experienced vascular surgeon (AW). The evaluation included a visual- and tactile examination of the replica and a comparison between diameter measurements obtained from multiplanar reconstructions (MPR) of the CTA-images, using a postprocessing work station (Multimodality Workplace (MMWP), Siemens Medical Systems, Forchheim, Germany), with the corresponding measures from the 3D-CAD-model and the physical RP-model. Catheterisation of and stent placement in the model was done under fluoroscopic guidance (Multistar, Siemens Medical Systems, Forchheim. Germany) with the following parameters: $70 \mathrm{kV}$, $300 \mathrm{mAs}$ and $2 \mathrm{mGy}$.

\section{Results}

The aortic inner wall with and without calcified plaque and the corresponding final RP-model of the aortic lumen are displayed in figure 2. The figure also shows a segment of the aorta where calcified plaque has reduced the inner diameter by $40 \%$. The entire 3D CAD-model, printed plaster model and silicon replica of the aorta are displayed in Fig. $3 a-c$.

The median (interquartile range) difference between diameters obtained from CTA- and RP plaster-model at 19 predefined locations was $0.5 \mathrm{~mm}$ 


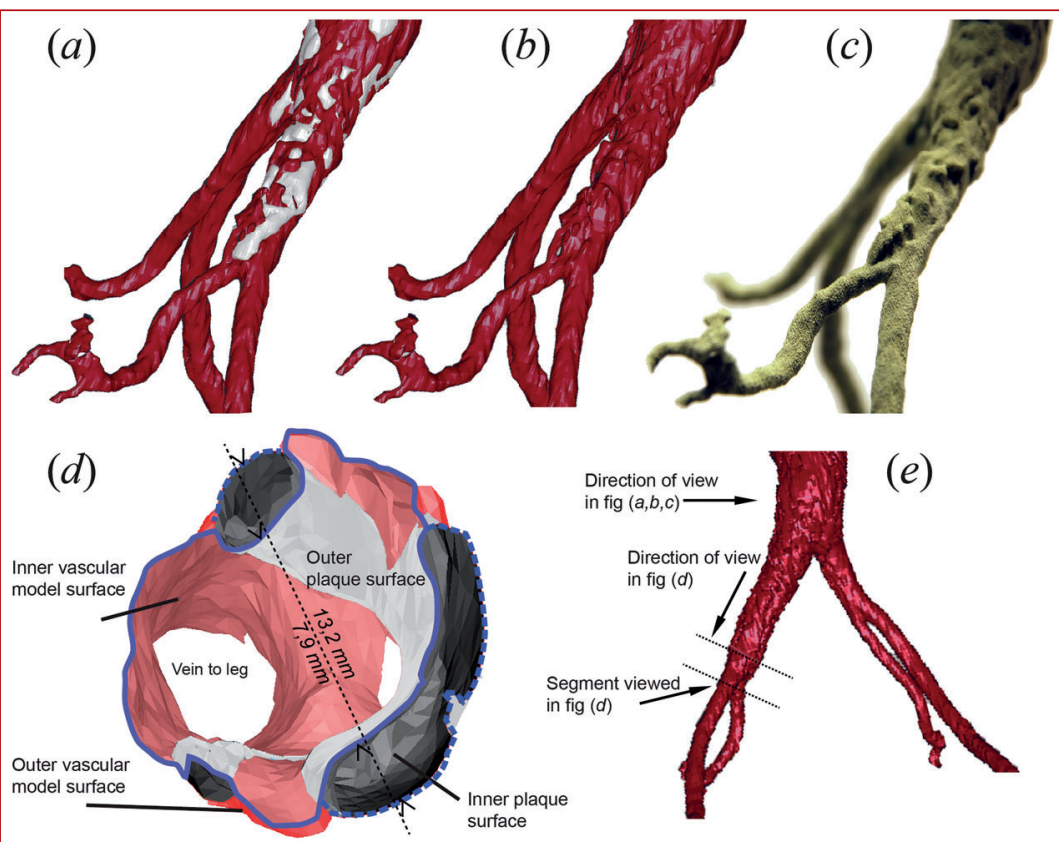

Figure 2: $(a)$ : CAD model of the artery inner wall with calcified plaque (displayed as white areas). (b): CAD model of the artery inner wall without calcified plaque (lumen). (c): Plaster model of the artery inner wall without calcified plaque (true lumen). (d): Cross section of artery showing the influence of calcified plaque on the diameter. $(e)$ : Figure showing the positions and direction of view of figure $(a)-(d)$.

$(1 \mathrm{~mm})$ which corresponds to a relative median difference of $4.6 \%$ (7.0\%). The average wall thickness of the silicone model was $3.5 \mathrm{~mm}$.

The elastic property and performance during stent implantation was considered good by the vascular surgeon (Fig. 4). Due to the rough surface of the RP model, the transparency of the silicone model was not optimal, although acceptable (Fig. 3f). The duration of time and the cost for creating the model were approximately 18 working hours and 1900 EUR.

\section{Discussion}

This paper describes a method for creating a silicone replica of an aorta by using CTA-data and RP. The two main issues addressed in this report were how to remove the calcified plaque related transition area from the lumen and how to create a life size physical model of the aorta.

During the detailed process planning the importance of atherosclerotic plaque contribution to the inner wall geometry became apparent. Failure to distinguish atherosclerotic plaque from the contrast enhanced lumen during postprocessing of the CTAimages causes the final reconstruction to have a falsely large inner wall diameter. This problem is not trivial since atherosclerosis is a common pathology in aneurysmal aortas. When removing the calcified plaque contribution from the lumen-mask one have to consider the transition area in order to obtain a correct inner wall representation. Commercially available postprocessing software are, unfortunately, unable to automatically do that.
Although, the multi-step editingmanoeuvre described in the present report resulted in a highly accurate $3 \mathrm{D}$-model of the true inner lumen, it has some obvious limitations. The method is labour intensive and a profound knowledge in advanced postprocessing is required. Furthermore, the optimal threshold attenuation level for anatomical structures is difficult to define and the final result is therefore highly operator dependent [15]. To overcome this limitation the final 3D-CAD-model should be carefully reviewed against the CTA-images before the RP process is initiated. In the present report the CTA- measurements were done in MPR-mode, allowing the operator to obtain the corresponding plastermodel diameters. Segmentation error may thereby have contributed to the observed differences.

Initial experiences on automatic calcified plaque removal using dualenergy CTA technology have shown promising results, with a high sensitivity for the detection of relevant stenosis. However, high-grade stenosis was overestimated [19] and plaque subtraction less accurate in small arteries [13]. Nevertheless, the technique is time efficient, easy to interpret and reduces the need for advanced postprocessing, and has the potential to become the golden standard for providing true CTA luminogram.

There are several different RP-methods available and one problem is to find the method most suitable for a specific application [8, 9]. Every method has its own pros and cons, e.g. different materials and different accuracy [22]. A number of RP methods where investigated and the main reasons for choosing the $\mathrm{Z}$ Corp machine where lower cost, faster printing time and the possibility to easily remove the plaster model from the silicon [11]. A drawback of the RP-machine used in the present 
(a)
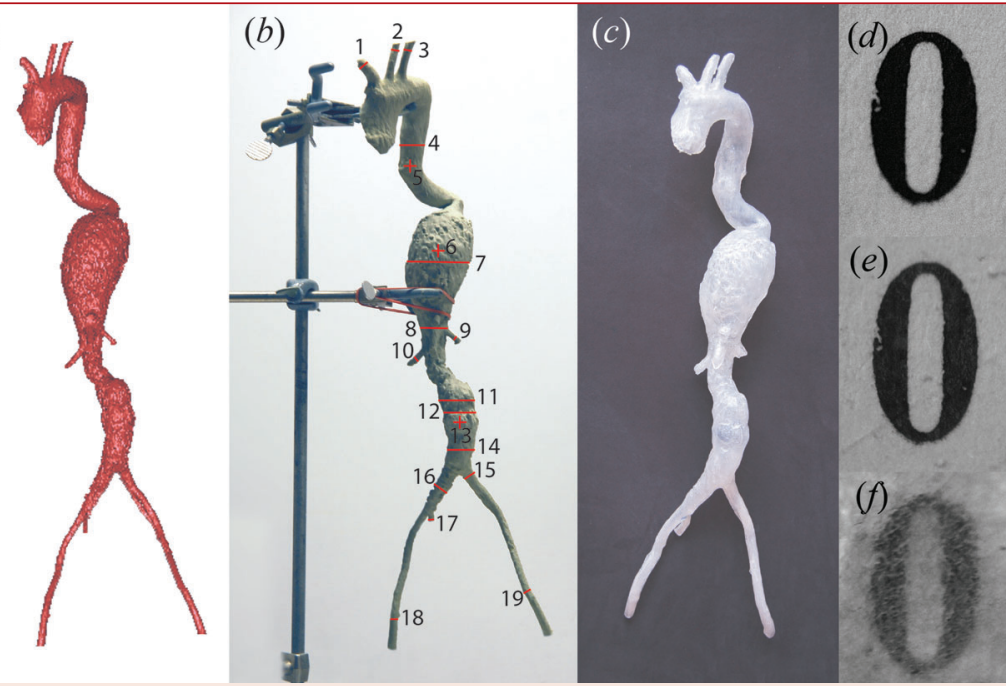

Figure 3: $(a)$ : 3D CAD model. (b): Printed plaster model. (c): Silicon model. $(d)$ : Photo of digit printed on a white paper. $(e)$ : Photo of digit displayed behind silicon cast on a smooth surface. $(d)$ : Photo of digit displayed behind the wall of the silicon replica of the aorta.

Table I: Diameters obtained from RP plaster-model and CT-angio at 19 predefined locations defined in figure $3 b$.

\begin{tabular}{|rrr|}
\hline Measuring location & Plaster diameter $(\mathrm{mm})$ & CT-angio diameter $(\mathrm{mm})$ \\
\hline 1 & 9.8 & 9.7 \\
2 & 7.1 & 6.7 \\
3 & 8.4 & 7.9 \\
4 & 24.5 & 24.7 \\
5 & 23.7 & 22.4 \\
6 & 58.5 & 57.7 \\
7 & 58.2 & 58.7 \\
8 & 27.0 & 27.1 \\
9 & 4.6 & 4.5 \\
10 & 6.8 & 5.9 \\
11 & 34.6 & 30.0 \\
12 & 29.8 & 29.6 \\
13 & 31.6 & 30.9 \\
14 & 25.7 & 23.7 \\
15 & 11.2 & 10.1 \\
16 & 14.5 & 13.4 \\
17 & 5.7 & 5.6 \\
18 & 7.8 & 7.0 \\
19 & 7.2 & 7.1 \\
\hline
\end{tabular}

report is the limited fabrication size. The CAD-model had to be sliced in three parts to fit the machine, which may add uncertainty to the accuracy of the final full size model. The sealing of the surface adds extra thickness to the model. This contribution was, however, considered insignificant.

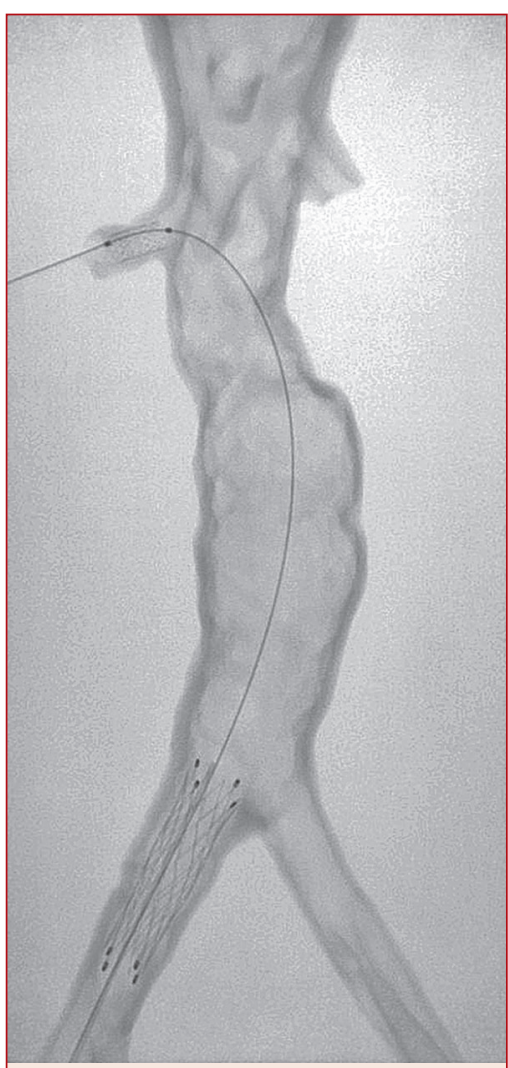

Figure 4: Fluoroscopy of the model showing a .014 " guidewire from the right femoral artery trough the right renal artery. A self expandable stentgraft has been deployed in the right common iliac artery and a balloon expandable stent in the right renal artery.

Due to the complexity of the aortic geometry the application of silicone to the RP-model of the lumen was made manually, layer by layer. An advantage with this is that no cavity mould is needed which reduces printing time and costs. It could be argued that by applying the silicone manually, a correct wall thickness of the silicone model is difficult to achieve. The accuracy of the wall thickness of the silicone model was, however, considered less important than the accuracy of the inner wall configuration. 
The final replica had a sufficient strength and flexibility to mimic the natural vessel. Model-based endovascular simulation allowed reproduction of comprehensive endovascular process, including realistic tactic feel for catheterization and stent implantation as well as fluoroscopic imaging in a Cath-lab. The rough surface of the RP-model resulted in a slightly impaired transparency of the siliconmodel. To enhance the transparency the RP-model should be coated and polished, before the silicon is applied (Fig. 3d-f).

The importance of plaque removal depends on the purpose of the model and on the atherosclerotic burden. The main value of an exact replica, as described in the present report, is for planning a complex endovascular custom-made stentgraft implantation. In such instances, the inner wall configuration of the aorta, as well as of the branches, should be thoroughly reviewed and the model should be adjusted accordingly. This issue is, however, seldom (or never) addressed in the literature. A less accurate model, and thereby less costly and work intensive, would do for general interventional training. Regardless of the objective it is important to obtain an aortic-wall alike material properties.

\section{Conclusions}

The integration of RP-techniques with CAD-based reconstruction of 3D-medical imaging data provides the needed tools for making a truly patient specific replica of the whole aorta with high accuracy. Calcified plaque removal is necessary to achieve an accurate inner wall configuration.

\section{Acknowledgement}

The authors would like to acknowledge Materialize Group in Belgium for their assistance when extracting the CAD-model.

\section{Conflicts of interest}

There are no conflicts of interest existing.

\section{References}

1 Armillotta A, Bonhoeffer P, Dubini G, Ferragina S, Migliavacca F, Sala G, Schievano S. Use of rapid prototyping models in the planning of percutaneous pulmonary stent implantation, J Engineering in Medicine 2007; 221 (4): $407-16$.

2 Chong CK, How TV, Black RA, Shortland AP, Harris PL. Development of a Simulator for Endovascular Repair of Abdominal Aortic Aneurysms. Ann Biomed Engineering 1998; 26: 798 - 802.

3 Chua CK, Leong KF, Lim CS. Rapid Prototyping - Principles and Applications, 2003, World Scientific Publishing Co. Pte. Ltd.

4 Dobrin, PB. Animal Models of Aneurysms, Ann Vasc Surg 1999; 13 (6): $641-8$.

5 Erickson DM, Chance D, Schmitt S, Mathis J. An opinion survey of reported benefits from the use of stereolithographic models. J Oral Maxillofac Surg 1999; 57 (9): 1040 - 3.

6 Golesworthy, T. To the heart of the matter - CRDM employs rapid manufacturing for a cardio vascular implant, Time Compression Technologies 2010; 18 (3): 45.

7 Hernandez-Hoyos M, Orkisz M, Puech P, Mansard-Desbleds C, Douek P, Magnin IE. Computer-assisted analysis of three-dimensional MR angiograms. Radiographics 2002; 22 (2): $421-36$.
8 Hieu LC, Zlatov N, Vander Sloten J, Bohez E, Khanh L, Binh PH, Oris P, Toshev Y. Medical rapid prototyping applications and methods, Assembly Automation 2005; 25 (4): 284-92.

9 Håkansson A \& Lundberg J. A Systematic Evaluation of RT Methods for SME's, Time Compression Technologies 2004 Vol. 12, No 4, Rapid News Publications plc.

10 Knox K, Kerber CW, Singel SA, Bailey MJ, Imbesi SG. Rapid prototyping to create vascular replicas from CT scan data: making tools to teach, rehearse, and choose treatment strategies. Catheter Cardiovasc Interv 2005; 65 (1): 47-53.

11 Lermusiaux P, Leroux C, Tasse JC, Gastellani L, Martinez R. Aortic Aneurysm: Construction of a Life-size Model by Rapid Prototyping, Ann Vasc Surg 2001; 15 (2): 131.

12 Markl M, Schumacher R, Kuffer J, Bley TA, Hennig J. Rapid vessel prototyping: vascular modeling using 3 t magnetic resonance angiography and rapid prototyping technology. Magma 2005; 18 (6): 288-92.

13 Meyer BC, Werncke T, Hopfenmüller W, Raatschen HJ, Wolf KJ, Albrecht T. Dual Energy CT of Peripheral Arteries: Effect of Automatic Bone and Plaque Removal on Image Quality and Grading Stenosis. Eur J Radiol 2008; 68 (3): 414-22.

14 Milovanovic J, Trajanovic M. Medical Applications of Rapid Prototyping, Mechanical Enginnering 2007; 5 (1): $79-85$.

15 Petzold R, Zeilhofer HF, Kalender WA. Rapid prototyping technology in medicine - basics and applications, Comput Med Imaging Graph 1999; 39: $277-84$.

16 Radecka E, Brehmer M, Holmgren K, Palm G, Magnusson P, Magnusson A. Pelvicaliceal Biomodeling as an Aid to Achieving Optimal Access in Percutaneous Nephrolithotripsy, J Endourology 2006; 20 (2): $92-101$. 
17 Raghavan ML, Kratzberg J, de Tolosa EMC, Hanaoka MM, Walker P, da Silva ES. Regional distribution of wall thickness and failure properties of human abdominal aortic aneurysm, J Biomech 2006; 39: 3010 - 6.

18 Sulaiman A, Boussel L, Taconnet F, Serfaty J.M, Alsaid H, Attia C, Huet L, Douek P. In vitro non-rigid lifesize model of aortic arch aneurysm for endovascular prosthesis assessment, Eur J Cardio-thoracic Surg, 2008; 33: $53-7$.

19 Thomas C, Korn A, Ketelsen, D, Danz S, Tsifikas I, Claussen CD, Ernemann U, Heuschmid M. Automatic lumen segmentation in calcified plaques: dual-energy CT versus standard reconstructions in comparison with digital subtraction angiography. AJR Am J Roentgenol 2010; 194 (6): 1590 - 5.

20 Wetzel SG, Ohta M, Handa A, Auer J-M, Lylyk P, Lovblad K-O, Babic D, Rufenacht D.A. From Patient to Model: Stereolithographic Modeling of the Cerebral Vasculature Based on Rotational Angiography, Am J Neuroradiol 26: 1425-1427, June/July 2005.

21 Winder RJ, Sun Z, Kelly B, Ellis PK, Hirst D. Abdominal aortic aneurysm and stent graft phantom manufactured by medical rapid prototyping. J Med Eng Technol 2002; 26 (2): $75-8$.

22 Wohlers T. Future potential of rapid prototyping and manufacturing around the world, Rapid Prototyping Journal 1995, 1 (1): 4- 10.
23 Wurm G, Tomancok B, Pogady P, Holl K, Trenkler J. Cerebrovascular stereolithographic biomodeling for aneurysm surgery, J Neurosurg 2004, 100: 139-45.

\section{Correspondence address}

Dr. Anders Wanhainen, MD, PhD

Department of Surgical Sciences Section of Vascular Surgery Uppsala University Uppsala University Hospital SE-75285 Uppsala Sweden

E-mail: andwan@algonet.se

Submitted: 28.02.2011

Accepted after revision: 13.04.2011 